\title{
Empyema thoracis: a role for open thoracotomy and decortication
}

\author{
J A Carey, J R L Hamilton, D A Spencer, K Gould, A Hasan
}

\begin{abstract}
Background-Thoracentesis and antibiotics remain the cornerstones of treatment in stage I empyema. The management of disease progression or late presentation is controversial. Open thoracotomy and decortication is perceived to be synonymous with protracted recovery and prolonged hospitalisation. Advocates of thoracoscopic adhesiolysis cite earlier chest drain removal and hospital discharge. This paper challenges traditional prejudice towards open surgery.
\end{abstract}

Methods-A five year audit of empyema cases referred to a regional cardiothoracic surgical unit analysing previous clinical course, surgical management, and outcome.

Results-Between February 1992 and February 1997 , the number of referrals to this centre increased dramatically. Twenty two children were referred for surgery (15 boys, seven girls; age range, 0.5-16 years). Before referral, patients had been unwell for 6-50 days (median, 15), had been treated with several antibiotics, and had undergone chest ultrasound (15 patients), computed tomography (five patients), pleural aspiration attempts (13 patients), and intercostal drainage (seven patients). The organism responsible was identified in only two cases (Streptococcus pneumoniae). Three patients had intraparenchymal abscess formation. Eighteen patients underwent open thoracotomy and decortication. Drain removal was performed on the first or second day. Fever resolved within 48 hours. Median hospital stay was four days. All patients had complete clinical and radiological resolution.

Conclusions-Treatment must be tailored to the disease stage. In stage II and III diseases, open decortication followed by early drain removal results in rapid symptomatic recovery, early hospital discharge, and complete resolution. In the early fibrinopurulent phase, alternative strategies should be considered. However, even in ideal cases, neither fibrinolysis nor thoracoscopic adhesiolysis can achieve more rapid resolution at lower risk.

(Arch Dis Child 1998;79:510-513)

Keywords: empyema; decortication

Empyema is a rare complication of community acquired pneumonia in children. Diagnostic and staging criteria have been described. ${ }^{12}$
Thoracentesis and antibiotic treatment remain the cornerstones of treatment for stage I disease. The management of disease progression or late presentation is controversial. Open thoracotomy and decortication have been perceived to be synonymous with protracted recovery and prolonged hospitalisation. Advocates of thoracoscopic adhesiolysis cite earlier chest drain removal (median, 7 days) and hospital discharge (median, 8 days). ${ }^{3-6}$ The purpose of our paper is to emphasise the need to tailor the treatment modality to the stage of disease, to encourage early surgical referral, and to challenge traditional prejudice towards open surgery.

\section{Methods}

An analysis of all admissions to the hospitals within the Northern Health Region over a seven year period was performed to determine the yearly prevalence of pneumonia, pleural effusion, and parapneumonic empyema in children under the age of 16 years. Using international disease classification codes, a regional database was searched for community acquired pneumonia cases admitted to these hospitals, excluding post-traumatic and tuberculous groups.

In a consecutive series of childhood empyema referrals to a regional cardiothoracic surgical centre, patterns of referral, previous clinical course, stage of disease at referral, subsequent surgical management, and outcome were examined.

MANAGEMENT PROTOCOL

Patients were transferred to the surgical unit, had chest radiographic and ultrasound examinations, and were transferred to the operating theatre on the day of referral. The patients were anaesthetised and underwent rigid bronchoscopy to: exclude the presence of a foreign body or structural abnormality in the airway; perform bronchial lavage; and obtain specimens for microbiological examination. Treatment was based on empyema stage. In stage I disease, when ultrasound examination identified anechoic collections and absent loculation, needle aspiration confirmed the presence of fluid and an intercostal drain was positioned. Early stage II disease (fibrinous septation, absence of homogenous echogenic loculations or thickened parietal rind on ultrasound) was evaluated for video assisted thoracoscopic adhesiolysis by needle aspiration, followed by creation of a single port and thoracoscope insertion. Late stage II disease and stage III disease were treated by open thoracotomy and decortication. The aims of surgery were to per- 
form a complete debridement and to achieve full lung re-expansion without air leaks or residual spaces. A limited lateral thoracotomy was performed. Initially, the parietal pleura was not breached. Separation of the pleura from the chest wall and diaphragm was achieved by blunt dissection. The thickened parietal pleura was then incised. The plane between the parietal pleura and the underlying encased lung was dissected. The thickened parietal pleura was then excised. The thick custard-like fibinopurulent debris was completely removed and the visceral peel was stripped to release the entrapped lung. Parenchymal lung abscesses were treated by deroofing, debridement, and by laying an intercostal muscle bundle over exposed bronchi. The chest wall was then closed in layers after the insertion of one or two intercostal drains. The drains were placed on continuous low suction $(2-4 \mathrm{kPa})$. Older children were extubated on the operating table. Otherwise, the children were transferred to intensive care and extubated the same evening or next morning. Chest radiography was performed immediately after surgery to ensure complete expansion. In a minority of cases, there was a small air leak. These patients were taken off suction, provided the lung was expanded. Chest radiography was repeated the next day, before chest drain removal. Early drain removal, adequate pain control, intensive physiotherapy, and early mobilisation were the key factors in achieving prompt hospital discharge. Analgesia was achieved by a combination of a paravertebral marcaine block given before thoracotomy and postoperative oral medications. When specific organisms had not been identified, the children were treated empirically with antibiotics for two weeks. All children were reviewed in the outpatient clinic at four weeks with repeat chest radiography.

\section{Results}

REGIONAL DATA

Between January 1989 and December 1996, the yearly admission rates for community acquired pneumonia remained unchanged and the prevalence of both parapneumonic effusions $(3 \%)$ and parapneumonic empyema $(0.9 \%)$ remained constant. During this period, $11 \%$ of empyema cases underwent decortication.

SURGICAL REFERRALS

Between February 1992 and February 1997, 22 patients were referred to our surgical unit. Up until 1995, there were very few surgical referrals each year, but in later years, the referral rate escalated. Median age was 7 years (range, 0.5-16). There were 15 boys and seven girls. The median duration of illness before referral was 15 days (range, 6-50). Presentation to the general practitioner had taken place at 1-14 days after start of the illness. Hospital referral had occurred at 1-11 days after presentation. Patients had been hospitalised for a median duration of 7 days (range, 1-48 days) before transfer to the regional cardiothoracic surgical centre.
Before surgical referral, individual patients had been administered between three and seven antibiotic agents including penicillin derivatives, cephalosporins, aminoglycosides, macrolides, and metronidazole. Treatment had commenced blindly. Pleural aspiration attempts had been made in 13 patients after commencement of antibiotic treatment. The organism responsible had been identified in only two patients. Both had pneumococcal infection. Intercostal drainage had been undertaken in seven patients. Fifteen of the patients had undergone chest ultrasound examination and five patients had undergone computed tomography.

At the time of surgical referral, anaemia, leucocytosis, fever $\left(>38^{\circ} \mathrm{C}\right)$, and weight loss were noted in most patients. One child had an immunological deficiency ( $\operatorname{IgG}_{2}$ absence). Three patients had a pleural exudate and absent septation (stage I disease). One patient had fibrinous septation and minimal rind formation (stage II disease). Eighteen patients had organised, multiloculated empyema with lung entrapment (stage III disease). Three of the patients with organised collections had intraparenchymal lung abscess formation (stage IIIc disease). It is only recently that children with stage I and II diseases have been referred for assessment and treatment.

\section{SURGICAL MANAGEMENT}

At bronchoscopy, no foreign bodies were found. Three patients with stage I disease were treated by intercostal drain insertion alone. The site was determined by prior ultrasound examination. One patient with early stage II disease underwent video assisted thoracoscopic adhesiolyis (using three port sites) and insertion of a single intercostal drain. The remaining 18 patients underwent open thoracotomy and decortication. Three patients with a lung abscess underwent deroofing and debridement of the abscess. In two cases, an intercostal muscle flap was applied to the underlying open bronchi. In one case, a strip of pericardium was used to reduce the air leak.

SURGICAL RESULTS

There were no complications. The removal of intercostal drains was accomplished within 48 hours in all patients. The drain was removed within 24 hours in 16 patients. Fever resolved within 48 hours. Median hospital stay was 4 days (range, 3-7). The organisms responsible remained unidentified. In two cases, pneumococcal antigen was identified in purulent fluid obtained at the time of surgery. No organisms were grown from any intraoperative specimen. Virological screening was also negative. Patients were seen in outpatient clinics at four weeks. All patients had complete clinical and radiological resolution.

\section{Discussion}

The immediate objectives in the treatment of empyema are to eradicate persistent fever, to evacuate pleural contents, and to fully reexpand the lung. The long term objective is to prevent chronic lung damage. Simple para- 
pneumonic effusions (>10 mm) should be differentiated from empyema by aspiration and fluid analysis. ${ }^{2}$ Staging initially involves plain chest radiographs (including lateral and decubitus views), chest ultrasound, and occasionally, computed tomography. Disease stage determines the choice of intervention.

In stage I disease, antibiotic treatment is usually effective. Failure to identify the responsible organism, blind antibiotic selection, and inadequate dosage are important issues that need to be considered if treatment fails. Serial radiographs and ultrasound evaluation should be used to identify disease progression after commencement of antibiotic treatment. Clinical features of toxicity, the presence of a large effusion, progressive fluid accumulation, incomplete aspiration, or early septation are indications for drainage. A timely, well positioned pigtail catheter or intercostal chest tube will provide adequate drainage and prevent disease progression. The choice of catheter or chest tube and its positioning should be guided by ultrasound examination.

If the disease has presented with, or progressed to, the formation of multiloculated collections and fibrinopurulent exudate (stage II), fibrinolysis, video assisted thoracoscopic adhesiolysis, or open surgery should be considered. There are no controlled trials in paediatric empyema comparing alternative interventions. However, the excellent results achieved after open surgery in our series provide a valuable benchmark against which the prospective outcome of alternative interventions should be judged.

When considering alternatives to open surgery, it should be pointed out that it is only possible to achieve comparable results in the earliest phases of stage II disease. Radiological features predict the likelihood of resolution and the prospect of protracted treatment using alternative treatment modalities. Inappropriate fibrinolysis and video assisted thoracoscopic adhesiolysis should be avoided and surgical intervention should not be delayed.

In early stage II disease, fibrinolysis will be most effective in cases where simple video assisted thoracoscopic adhesiolysis is possible. Even then, recovery and hospital discharge after this treatment will be more rapid because of earlier removal of the intercostal drains. A protracted hospital stay involving insertion of multiple intercostal chest drains and extended fibrinolytic treatment is not acceptable when rapid resolution is attainable with alternative safe treatment modalities, especially in children who have been febrile and anorexic for some time. The cost of extended fibrinolytic treatment is also a consideration.

In intermediate stage II disease, video assisted thoracoscopic clearance might be possible. This is a judgment that must be made at surgery. If complete clearance and lung reexpansion is not possible by video assisted thoracoscopic techniques, then protracted intercostal drainage, instillation of fibrinolytics for drain blockages or residual pleural collections, and late conversion to open thoracotomy are unacceptable scenarios. However, even in ideal

\section{Key messages}

- Management principles: treatment must be based on radiological staging and clinical status

- Antibiotics and drainage are usually successful in stage I disease

- Factors contributing to development of stage II and stage III disease: late presentation, failure to identify responsible organisms, inadequate antibiotic treatment, reluctance to drain, suboptimal drain placement, under use of ultrasound imaging for initial staging and assessment of treatment response, and late surgical referral

- Surgical results: in stage II and stage III empyema, open thoracotomy and decortication is followed by drain removal within 48 hours, prompt hospital discharge, and complete resolution. Using these results as a benchmark, alternative treatments should only be undertaken in carefully selected patients

candidates, video assisted thoracoscopic techniques cannot result in earlier chest drain removal, mobilisation, or hospital discharge than open surgery. The operative time for open decortication will often be less than for video assisted thoracoscopic adhesiolysis. The combined length of the scar for the video assisted thoracoscopic ports is not much less than that of a limited thoracotomy. There is no reduction in operative risk. A prospective comparison of postoperative pain and long term sequelae has not been undertaken in this subgroup. However, after open surgery, postoperative discomfort is easily managed if the intercostal drain is removed early. Therefore, the reality is that pain relief is not a clinical problem. Furthermore, there are no significant differences in lung function at follow up between children who have undergone simple tube drainage or thoracotomy. ${ }^{78}$

In advanced stage II disease, the decision to perform open surgery is clear at the time of surgery. At this stage, the fibrinopurulent material becomes dense and begins to organise; the rind is forming; and the pleural cavity is often multiloculated. These changes are apparent on ultrasound and computed tomography imaging. At surgery, needle aspiration results in a dry tap or minimal fluid extraction. Fibrinolysis will be ineffective. Clearance of the pleural cavity and lung re-expansion are impossible by thoracoscopic intervention.

When stage III disease has developed, fibrinolysis and video assisted thoracoscopic adhesiolysis have no role. There are no tenable arguments for delaying or avoiding open decortication. Open surgery should be undertaken in the confidence that meticulous technique results in early drain removal, rapid recovery, prompt hospital discharge, and complete resolution.

In conclusion, treatment must be tailored to disease stage. We advocate precise radiological 
staging at presentation, thoracentesis, vigilance following commencement of appropriate antibiotic treatment, and early referral of those patients who fail to respond to avoid open thoracotomy. Children who present with stage II or III diseases should be assessed and treated by a multidisciplinary team at the regional cardiothoracic surgical centre. In stage II and III diseases, open decortication followed by early drain removal results in rapid symptomatic recovery, early hospital discharge, and complete resolution. In the early fibrinopurulent phase, alternative strategies should be considered. However, even in ideal cases, neither fibrinolysis nor thoracoscopic adhesiolysis can achieve more rapid resolution at lower risk. The benchmark is set by the results achieved with open thoracotomy and decortication.
The authors are grateful for the invaluable contribution made by M Mythen, Patient Information Systems, Freeman Hospital, and the secretarial assistance provided by L Parker and K Pinnock.

1 American Thoracic Society. Management of nontuberculous empyema. Am Rev Respir Dis 1962;85:935-6. 2 Light RW. A new classification of parapneumonic effusions and empyema. Chest 1995;108;299-301.

3 Kern JA, Rodgers BM. Thoracoscopy in the management of empyema in children. $f$ Ped Surg 1993;28:1128-32.

4 Stovroff M, Teague G, Parker P, Ricketts RR. Thoracoscopy in the management of pediatric empyema. 9 Ped Surg 1995; in the manag

5 Silen ML, Weber TR. Thoracoscopic debridement of loculated empyema thoracis in children. Ann Thorac Surg 1995; 59:1166-8.

6 Davidoff AM, Hebra A, Kerr J, Stafford PW. Thoracoscopic management of empyema in children. $\mathcal{F}$ Laparoendosc Surg 1996;6(suppl 1):S51-4.

7 Mayo P, Saha SP. Acute empyema in children treated by open thoracotomy and decortication. Ann Thorac Surg 1982;34:401-7.

8 Hoff SJ, Neblett WW, Edwards KM, et al. Parapneumonic empyema in children: decortication hastens recovery in patients with severe pleural infections. Pediatr Infect Dis $\mathcal{F}$ 